\title{
Genetically engineered Newcastle disease virus expressing human interferon- $\lambda 1$ induces apoptosis in gastric adenocarcinoma cells and modulates the Th1/Th2 immune response
}

\author{
XUEFENG BU ${ }^{1 *}$, MI LI $^{1,2^{*}}$, YINGHAI ZHAO ${ }^{2}$, SHA LIU $^{2}$, MUBIN WANG $^{2}$, JINYING GE $^{3}$, \\ ZHIGAO BU ${ }^{3}$ and YULAN YAN ${ }^{4}$
}

\begin{abstract}
${ }^{1}$ Department of General Surgery, Affiliated People's Hospital of Jiangsu University; ${ }^{2}$ Clinical Medicine College of Jiangsu University, Zhenjiang, Jiangsu 212002; ${ }^{3}$ Key Laboratory of Veterinary Public Health of Ministry of Agriculture, State Key Laboratory of Veterinary Biotechnology, Harbin Veterinary Research Institute, Chinese Academy of Agricultural Sciences, Harbin, Heilongjiang 150001; ${ }^{4}$ Department of Internal Medicine, Affiliated People's Hospital of Jiangsu University, Zhenjiang, Jiangsu 212002, P.R. China
\end{abstract}

Received February 27, 2016; Accepted April 9, 2016

DOI: $10.3892 /$ or.2016.4925

\begin{abstract}
Interferon- $\lambda 1$ (IFN- $\lambda 1$ ), a recently discovered cytokine of the type III IFN family, was found to be a therapeutic alternative to type I IFN in terms of tumors. Using reverse genetics technique, we generated a recombinant Newcastle disease virus (NDV) LaSota strains named as human IFN- $\lambda 1$ recombinant adenovirus (rL-hIFN- $\lambda 1$ ) containing human IFN- $\lambda 1$ gene and further evaluated the expressing of IFN- $\lambda 1$ in human gastric adenocarcinoma cell line SGC-7901 after infected with rL-hIFN- $\lambda 1$ by using western blot analysis, RT-PCR and immunofluorescence analyses. IFN- $\lambda 1$ specific receptor IFNLR1 was detected on several gastric tumor cell lines including SGC-7901 and AGS and on PBMCs. The expression of the IFN- $\lambda 1$ proteins reached a high level detected in the supernatant harvested $24 \mathrm{~h}$ after the infection of tumor cells. The proliferation changes of SGC infected with rL-hIFN- $\lambda 1$ was significantly inhibited compared with NDV-infected group. Apoptosis was significantly induced by rL-hIFN- $\lambda 1$ in gastric cancer cells compared with NDV virus tested by TUNEL assay, western blot analysis and Annexin V flow cytometry. Due to the high dose of IFN- $\lambda 1$ expressed by the rL-hIFN- $\lambda 1$-infected tumor cells, the immune study showed that rL-hIFN- $\lambda 1$ increased IFN- $\gamma$ production [the T helper cell subtype 1 (Th1) response] and inhibited interleukin (IL)-13 production [the $\mathrm{T}$ helper cell
\end{abstract}

Correspondence to: Dr Yu-Lan Yan, Department of Internal Medicine, Affiliated People's Hospital of Jiangsu University, 8 Dianli Road, Zhenjiang, Jiangsu 212002, P.R. China

E-mail: ylyan2005@163.com

*Contributed equally

Key words: recombinant Newcastle disease virus, interferon- $\lambda$, rapoptosis, $\mathrm{T}$ helper cell subtype $1 / \mathrm{T}$ helper cell subtype 2 subtype 2 (Th2) response] to change the Th1/Th2 response of tumor microenvironment which inhibited tumor growth. This study aims at building recombinant NDV rL-hIFN- $\lambda 1$ as an efficient antitumor agent.

\section{Introduction}

In recent decades, an integrated approach to cancer treatment that includes surgery, chemotherapy, radiotherapy, gene therapy and immune therapy, has become a reasonable therapeutic strategy (1). Oncolytic virotherapy using agents such as Newcastle disease virus (NDV) is one of the new biological strategies for gene and immune therapy and has been tested against many different cancers including gastric cancer, skin tumors and several solid cancers (2-4). NDV, which is a member of the Paramyxoviridae family, is a non-segmented negative-strand RNA virus (NNSV). The NDV genes encode six major structural proteins, including nucleoprotein (NP), phosphoprotein $(\mathrm{P})$, matrix protein $(\mathrm{M})$, fusion protein $(\mathrm{F})$, hemagglutinin-neuraminidase $(\mathrm{HN})$ and large $(\mathrm{L})$ RNA-dependent RNA polymerase, in the following order 3'-NP-P-M-F-HN-L-5'. Oncolysis, apoptosis and enhancing innate immunity are the key mechanisms capacitating NDV as an agent against malignant cells (5). NDV virus can be propagated in embryonated chicken eggs, harvested from the allantoic fluid, and quantified by hemagglutination, NDV has been actively developed and evaluated as a vaccine vector for the control of human and animal diseases, these characteristics made the NDV virus easy medium to obtain (6-9).

Interferon- $\lambda 1$ (IFN- $\lambda 1$ ), also known as interleukin (IL)-29, is a recently discovered cytokine of the type III IFN family $(10,11)$. It is thought to have biologic properties similar to the type I IFNs. Unlike IFN- $\alpha$, the receptor for IFN- $\lambda 1$ expresses on a limited number of normal cells including dendritic cells, T cells, and intestinal epithelial cells $(12,13)$. Leukemia cells and colon, prostate, pancreatic, lung, hepatoma, glioblastoma, and breast cancer cells have also been shown to express IFNLR1 receptor (14-18). It is widely accepted that 
cytokines associated with a T helper cell subtype 1 (Th1) exhibit tumour-suppressive activity, whereas $\mathrm{T}$ helper cell subtype 2 (Th2) cytokines can promote tumour progression (14,19-21). IFN- $\lambda 1$ primarily inhibits IL-13 production and gives rise to IFN- $\gamma$ production in PBMCs (22). IFN- $\lambda 1$ receptor IFNLR 1 expresses on both naive and memory $\mathrm{CD}^{+}$ $\mathrm{T}$ cells (23). It is also reported when cultured with IL-29 the cytokine tumor microenvironment is skewed towards a Th1 response that results in reducing tumor growth (24).

In the present study, we used an established reverse genetics system (8) to generate a recombinant NDV LaSota viruses named as human IFN- $\lambda 1$ recombinant adenovirus (rl-hIFN- $\lambda 1$ ), we incerted IFN- $\lambda 1$ gene into a lentogenic recombinant LaSota (NDV) genome at the position between the $\mathrm{P}$ and $\mathrm{M}$ genes. We believe that this new recombinant virus can express IFN- $\lambda 1$ protein stably allowing us to consider it to br a promising agent for cancer therapy. Our group studied the antitumor effect of the rL-hIFN- $\lambda 1$ on gastric adenocarcinoma cell lines compared with NDV. Furthermore, in this study we also investigated whether the rL-hIFN- $\lambda 1$ is capable of regulating the IFN- $\gamma$ secretion (the Th1 response) and the IL-13 secretion (the Th2 response) indicating the change of Th1/Th2 balance in the tumor microenvironment that results in inhibiting tumor growth.

\section{Materials and methods}

Cells and viruses. The BSR cells (Harbin Veterinary Research Institute) for virus rescue, and the human gastric adenocarcinoma cell lines SGC-7901 and AGS were purchased from the Cancer Cell Repository (Shanghai Cell Bank, Shanghai, China; 2010-02-20) and the American Type Culture Collection (ATCC; Rockville, MD, USA). Gastric adenocarcinoma cells SGC-7901 and AGS cells were cultured in Dulbecco's modified Eagle's medium (DMEM; Invitrogen, Carlsbad, CA, USA) containing $10 \%$ fetal bovine serum (FBS) at $37^{\circ} \mathrm{C}$ in a humidified $5 \% \mathrm{CO}_{2}$ incubator. Cell culture reagents were acquired from Gibco (Grand Island,NY,USA). When the cells reached 50-70\% confluence, they were infected with $\mathrm{LL}-\mathrm{hIFN}-\lambda 1$ or NDV for further study. The NDV LaSota strain and the recombiant viruses were propagated and titrated in 9-day-old SPF embryonated chicken eggs. The NDV strain LaSota, SPF eggs and anti-NDV serum was provided by the Harbin Veterinary Research Institute, Chinese Academy of Agricultural Sciences. Human PBMCs were isolated from peripheral blood of volunteer donor with density gradient centrifugation over Histopaque-1077 (SigmaAldrich, St. Louis, MO, USA). Cells were collected and washed twice in RPMI-1640 medium, and then were resuspended at a final density of $10^{6} / \mathrm{ml}$ in RPMI-1640 medium supplemented with $10 \%$ heat-inactivated fetal calf serum (FCS) both from Invitrogen. Con-A stimulations were performed in 24-well plates using $1 \times 10^{6}$ whole PBMCs with $5 \mu \mathrm{g} / \mathrm{ml}$ Con-A (SigmaAldrich) in a final volume of $2 \mathrm{ml}$.

Materials. 3-(4,5-dimethyl-2-thiazolyl)-2,5-diphenyl-2H-tetrazolium bromide (MTT) was purchased from Amresco (Solon, OH, USA). Hoechst 33342 were obtained from Sigma-Aldrich. All PCR primers were purchased from Shanghai Sangon Biological Engineering Technology and Services Co., Ltd. (Shanghai, China). Total RNA from viral or tumour cells were extracted with TRIzol reagent (Invitrogen). Anti-cleaved caspase-3 was from ImmunoWay Biotechnology (Newark, DE, USA). Anti-IFN- $\lambda 1$ was from Santa Cruz Biotechnology, Inc. (Santa Cruz, CA). Anti-IFN $\lambda$-R1 was from R\&D Systems, Inc. (Minneapolis, MN, USA). HRP-conjugated goat anti-rabbit, HRP-conjugated goat anti-mouse and FITC-conjugated goat anti-mouse antibodies were purchased from CWBio (Shanghai, China). HRP-conjugated goat anti-rabbit, HRP-conjugated goat anti-mouse, and FITC-conjugated goat anti-mouse antibodies were purchased from CWBio. HRP-conjugated rabbit anti-chicken antibody was from EarthOx Life Sciences (Millbrae, CA, USA), and Cy3-conjugated rabbit anti-chicken antibody was purchased from KPL, Inc. (Gaithersburg, MD, USA). The polyvinylidene difluoride (PVDF) membrane and Luminata were provided by Millipore (Billerica, MA, USA). Trypsin, and EDTA-2Na were offered by Gibco. A mitochondrial transmembrane potential analysis kit was from Nanjing KeyGen Biotech Co., Ltd. (Nanjing, China). All other supplies for cell culture were obtained from Corning Costar (Corning, NY, USA).

Construction of full-length NDV plasmid and virus rescue. To construct a full-length recombinant genomic cDNA. The cDNA of the IFN- $\lambda 1$ genes was amplified from a previous study (25). The primers for amplification of human IFN- $\lambda 1$ gene are shown in (Table I). These primers included the gene start and termination sequences of the NDV genome and the Kozak sequence. The amplified human IFN- $\lambda 1$ gene was inserted into plasmid pBluescriptII $\mathrm{Ks}(+)$ then tested with nucleotide sequence analysis, the newly recombinant plasmid was named as pBS. IFN- $\lambda 1$. The amplified human IFN- $\lambda 1$ gene was digested by $P m e I$ and then inserted into the NDV genome cDNA through a unique PmeI site in the P-M intergenic region at nucleotide position 3165 of the NDV genome (8) to build the new recombinant plasmid named pBRN.hIFN- $\lambda 1$ (Fig. 2).

The rescue of recombinant NDV viruses from cloned cDNA was as follows: BSR cells infected with A modified virus expressing T7 RNA polymerase at a multiplicity of infection (MOI) of 1.0 and then transfected with pBowing in a six-well plate with pBRN.hIFN- $\lambda 1$ together with 3 helper plasmids [pBS-NP $(0.4 \mu \mathrm{g})$, pBS-P $(0.2 \mu \mathrm{g})$, and pBS-L $(0.2 \mu \mathrm{g})]$. After $16 \mathrm{~h}$ of incubation at $37^{\circ} \mathrm{C}$, the medium was replaced with $2 \mathrm{ml}$ Opti-MEM (Invitrogen) containing $0.5 \mu \mathrm{g}$ of TPCK-trypsin and the cells were incubated for another 3 days at $37^{\circ} \mathrm{C}$. The supernatant was then inoculated into the allantoic cavities of 9-day-old embryonated SPF eggs. After $72 \mathrm{~h}$ of incubation at $37^{\circ} \mathrm{C}$, the allantoic fluid was harvested and the virus was identified by hemagglutination assay using $1 \%$ chicken red blood cells. The resultant recombinant viruses were designated as rL-hIFN- $\lambda 1$.

Growth characteristics of the recombinant viruses. Virus growth was determined both in cell culture and embryonated chicken eggs. Confluent monolayers of SGC cells were infected with rl, rL-hIFN- $\lambda 1$ in 96-well plates at a MOI of 1.0. Every 12-h post-infection, the cellular monolayers of SGC cells were harvested by freeze-thawing three times. Virus titers were determined as $50 \%$ tissue culture-infective dose $\left(\mathrm{TCID}_{50}\right)$. Two hundred microliters of the recombinant virus dilution containing $10^{4} \mathrm{TCID}_{50}$ per ml was inoculated into the allantoic cavity of 9-day-old embryonated chicken eggs. After three 
Table I. Primer sequences used in the study.

Primer sequence (5'-3')

\begin{tabular}{lll}
\cline { 2 - 3 } Gene name & \multicolumn{1}{c}{ Forward } & \multicolumn{1}{c}{ Reverse } \\
\hline IFN- $\lambda 1$ & GACTgtttaaacTTAGAAAAAATACGGGTA & GACTgtttaaactcaggtggactcagggtgggttg \\
& GAAgtgccaccatggctgcagcttggaccgt & \\
IFNLR1 & ACCTATTTTGTGGCCTATCAGAGCT & CGGCTCCACTTCAAAAAGGTAAT \\
NDV-PMEI & GGAAATCAGGAAAATCAAGCGCCT & AGAATCAAAGTACAGCCCAAT
\end{tabular}

IFN- $\lambda 1$, interferon- $\lambda 1$; NDV, Newcastle disease virus.

Table II. The AI of SGC cells infected by rL-hIFN- $\lambda 1$ and NDV.

\begin{tabular}{lccc}
\hline Group & AI $(\mathrm{n}=10)$ & F-value & P-value \\
\hline rL-hIFN- $\lambda 1$ & $0.278 \pm 0.043$ & & \\
NDV & $0.098 \pm 0.021$ & 236.45 & $<0.001$ \\
CON & $0.022 \pm 0.011$ & & \\
\hline
\end{tabular}

$\mathrm{AI}$, apoptotic index; rL-hIFN- $\lambda 1$, human interferon- $\lambda 1$ recombinant adenovirus; NDV, Newcastle disease virus.

days, the allantoic fluids of inoculated eggs were harvested and clarified by centrifugation at $1,000 \mathrm{x}$ g for $10 \mathrm{~min}$. Supernatants were used for titration as described above.

Immunofluorescence analysis. Tumour cells were cultured in 24-well plates for $24 \mathrm{~h}$ as previously described, infected with rL-hIFN- $\lambda 1$ at a MOI of 1.0 , treated with NDV and phosphate-buffered saline (PBS) as controls. Then, at 24-h post-infection, the cells were fixed in $4 \%$ paraformaldehyde at $4^{\circ} \mathrm{C}$ overnight, followed by immunofluorescence staining with antibodies against NDV, IFN- $\lambda 1$ and Hoechst 33342 staining. These stained SGC cells were monitored using immunofluorescence microscopy.

MTT analysis. To measure the proliferation level, the survial of cells was assessed with MTT with six replicates for NDV, rL-hIFN- $\lambda 1$ and PBS. At 12, 24, 36, 48, 60 and 72-h postinfection, cell survival was determined by incubating the cells with MTT. Absorbance at $490 \mathrm{~nm}$ was determined with ELISA microplate readers (BioTek Instruments, Inc., Winooski, VT, USA).

RNA isolation and RT-PCR analysis. Total RNA of virus or tumor cells was extracted from the cultured cells using TRIzol reagent. First-strand cDNA synthesis was performed using oligo(dT) primers and M-MLV reverse transcriptase. The primer set for the IFN- $\lambda 1$ gene and the IFNLR1 gene were shown in (Table I). All the procedures were based on manufacturer's instructions.

TUNEL assay. Cells were cultured in 24-well plates with slides and $10 \% \mathrm{FBS}$ DMEM at $37^{\circ} \mathrm{C}$ with $5 \% \mathrm{CO}_{2}$. Then, the SGC cells were infected with rL-hIFN- $\lambda 1$ and NDV within the logarithmic growth phase and fixed in $4 \%$ paraformaldehyde. After fixing, the cells were stained according to the manufacturer's instructions. The slides were observed and imaged under an optical microscope. The apoptotic index (AI) was calculated as the number of apoptotic cells/(the number of apoptotic cells + the number of non-apoptotic cells) x $100 \%$.

Enzyme-linked immunoabsorbent assay. Gastric adenocarcinoma cells in six-well plates were infected with NDV or rL-hIFN- $\lambda 1$ virus at MOI of 1.0. Then the supernatant was harvested at $0,6,12,24,36$ and 48 -h post-infection. The expression levels of IFN- $\lambda 1$ in the supernatant were measured by means of enzyme immune assay as described by human IFN- $\lambda 1$ platinum kit (eBioscience, San Diego, CA, USA). The OD450 of the samples were determined and plotted as a standard curve. The standard curve was generated by serially diluting the stock enzyme IFN- $\lambda 1$ in dilution buffer supplied by the kit.

To measure the cytokine protein, cytokine levels within supernatants was measured with ELISA. Antibodies for IFN- $\lambda 1$, and IFN- $\gamma$ were purchased from eBioscience and for IL-13 from R\&D Systems, Inc. Manufacturer's instructions were followed. Standards and culture supernatants were plated in triplicate.

Western blot assay. SGC and AGS cells with or without treatment were lysed in RIPA lysis buffer with a protease inhibitor cocktail (Santa Cruz Biotechnology, Inc.). The protein concentrations were measured using a BCA kit (Thermo Fisher Scientific, Inc., Waltham, MA, USA). An equal amount of protein from each sample was loaded onto a $10 \%$ polyacrylamide gel and separated by electrophoresis. Then, the proteins were transferred to PVDF membrane (Millipore, Temecula, CA, USA). The membranes were blocked for $1 \mathrm{~h}$ in $5 \% \mathrm{BSA}$, incubated with primary antibodies against specific proteins (i.e., caspase-3 for apoptosis, anti-NDV for infection, anti-hIFN- $\lambda l$ for expression of recombiant gene, anti-IFNLR1 for detection of IFN $\lambda$-1 receptor components and $\beta$-actin as a control) and then incubated with HRP-conjugated secondary antibodies. The protein bands were scanned using a Typhoon 9400 variable mode imager (Amersham Biosciences, Buckinghamshire, UK) and detected by Pierce ECL Plus substrate (Thermo Fisher Scientific, Inc.).

Flow cytometry assay using Annexin V. Annexin V/propidium iodide (PI) double-staining was used to detect membrane 


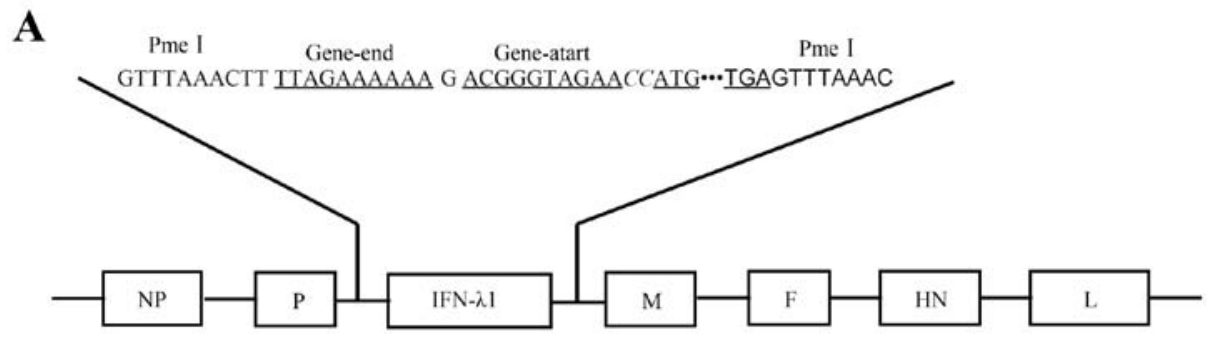

B

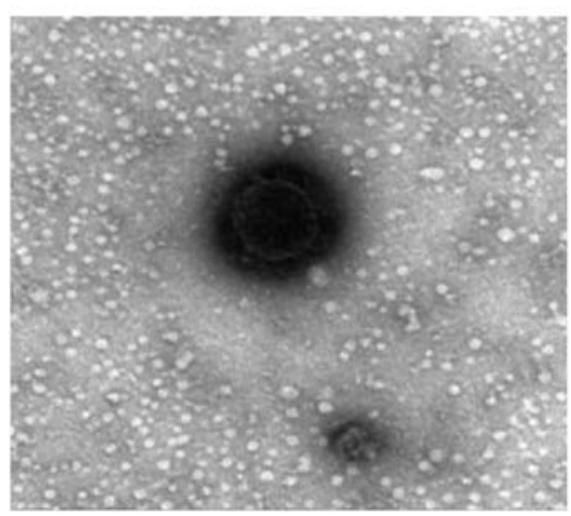

C

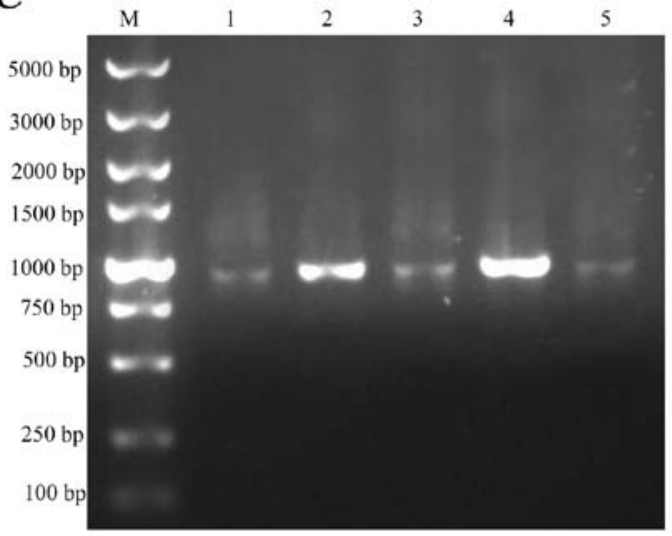

Figure 1. Generation of recombinant Newcastle disease virus (NDV) expressing the interferon- $\lambda 1$ (IFN- $\lambda 1$ ) gene. (A) Schematic representation showing the IFN- $\lambda 1$ gene was cloned into the intergenic region of NDV genome at the position between the P and M genes. The NDV gene start and the end signal sequences and the IFN- $\lambda 1$ sequences are boxed or underlined. (B) Electron microscopy of recombinant virus particles. NDV or rL-RVG propagated in eggs was prepared and partially purified by centrifugation through $20 \%$ sucrose. Viruses were stained with IgG purified from mouse naïve serum (AB, antibody), and with goat anti-mouse IgG conjugated with colloidal gold and then negatively stained. Transmission electron microscope showing two $200 \mathrm{~nm}$ conjugate human IFN- $\lambda 1$ recombinant adenovirus (rL-hIFN- $\lambda 1$ ) virus $(\rightarrow)$ at $80.0 \mathrm{kV}$. (C) Reverse transcription-polymerase chain reaction analysis of the presence of IFN- $\lambda 1$ in the recombinant virus rL-hIFN- $\lambda 1$. Lane M, DL5000 DNA marker; lanes 1-5, sequences around 1,000 dp cloned from the rescued viral total RNA and then amplified to sequence by nucleotide sequence analysis.

events according to the manufacturer's instructions (Biotech, Beijing, China). Flow cytometric analysis of labeled cells was performed using a FACSort flow cytometer (FACSCalibur) and data were analyzed using CellQuest software (both from BD Biosciences, Franklin Lakes, NJ, USA). The cytogram of the four quadrants in the figure was used to distinguish normal (Annexin $\mathrm{V}^{-} / \mathrm{PI}^{+}$), early apoptotic (Annexin $\mathrm{V}^{+} / \mathrm{PI}^{-}$), late apoptotic (Annexin $\mathrm{V}^{+} / \mathrm{PI}^{+}$), and necrotic cells (Annexin $\mathrm{V}^{-} / \mathrm{PI}^{+}$). The sum of early and late apoptosis was presented as total apoptosis. Finally, staining of the cells was observed with a fluorescence microscope. All experiments were carried out in triplicate.

Immunoelectron microscopy. Purified virus particles were bound to 200-mesh Formvar carbon-coated nickel grids (Electron Microscopy Science, Hatfield, PA, USA). For immunolabeling, grids were blocked in PBS containing $2 \%$ globulin-free BSA (Sigma-Aldrich) and incubated with chicken anti-NDV polyclonal IgG. Grids were then washed in blocking solution and incubated in goat anti-chicken IgG conjugated to 10-nm gold beads (Sigma-Aldrich). The grids received a final wash, followed by negative staining with $1 \%$ phosphotungstic acid. They were examined under a model H7500 transmission electron microscope (Hitachi High Technologies America, Inc., Schaumburg, IL, USA) at $80 \mathrm{kV}$. Images were obtained by using an XR100 digital camera system (Advanced Microscopy Techniques, Danvers, MA, USA).
Statistical analysis. The data comparisons were performed using one-way analysis of variance (ANOVA) in SPSS v17.0 software. $\mathrm{p}<0.05$ and $\mathrm{p}<0.01$ were considered statistically significant. All experiments were repeated at least three times.

\section{Results}

Generation of recombinant NDV LaSota strain expressing human IFN- $\lambda 1$ gene. Recombinant NDV expressing the human IFN- $\lambda 1$ was generated by inserting the IFN- $\lambda 1$ gene in the genomic cDNA of the NDV LaSota strain (Fig. 1A). For virus recovery, the heterogeneous gene carrying plasmid was transferred into 9-11 days old SPF embryonated chicken eggs for efficient virus propagation. After incubation for 5 days, the allantoic fluids were harvested and analyzed in a rapid plate HA test using chicken erythrocytes and observed by electron microscopy (Harbin Veterinary Research Institute) (Fig. 1B). The obtained viruses were named as $\mathrm{rL}-\mathrm{hIFN}-\lambda 1$. The presence and accuracy of the inserted IFN- $\lambda 1$ gene, the sequences from $\mathrm{rL}-\mathrm{hIFN}-\lambda 1$ genome were confirmed by nucleotide sequence analysis (data not shown), the sequences contained whole IFN- $\lambda 1$ genome were cloned by RT-PCR (Fig. 1C) by primers NDV-PMEI (Table I) set above and blow the PMEI set on NDV. The results showed they represented the correct sequences successfully inserted into the NDV genome, and mutation did not occur in rL-hIFN- $\lambda 1$. 

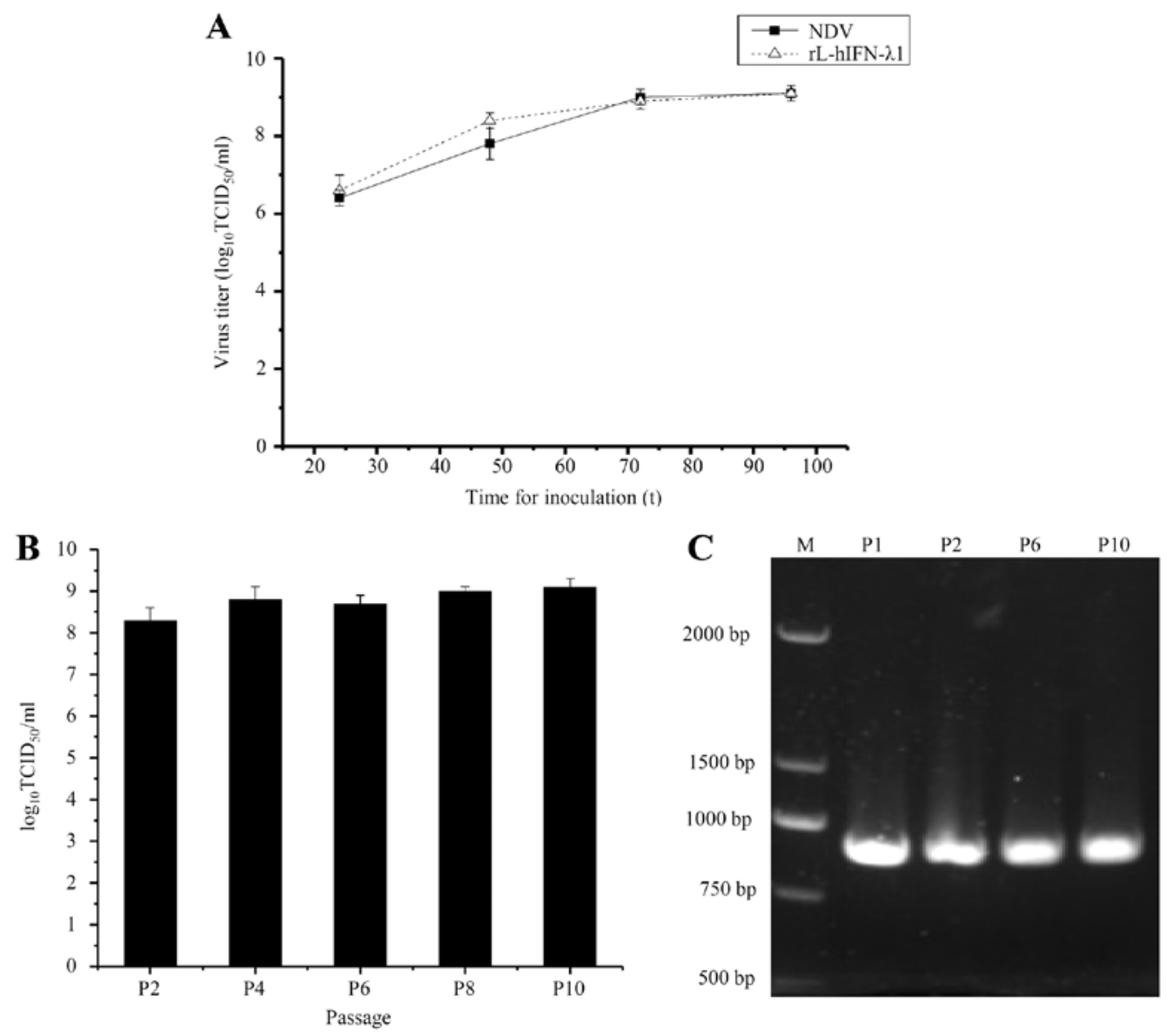

Figure 2. Growth characteristics for Newcastle disease virus (NDV) and human interferon- $\lambda 1$ recombinant adenovirus (rL-hIFN- $\lambda 1$ ). (A) The SGC cells were infected with NDV or rL-hIFN- $\lambda 1$ at a MOI of 0.01 . Every 24 -h post-infection, the cells were harvested. Virus titers at each time-point were determined by $\mathrm{TCID}_{50}$ titration in SGC cells. (B) The allantoic fluid of SPF eggs inoculated with $100 \mathrm{TCID}_{50}$ of recombinant virus were harvested after 4-day inculation. The amount of virus was determined by end-point titration. The results represented mean values from three independent experiments. (C) mRNA expression in some of the generation of rL-hIFN- $\lambda 1$ virus. Lane M, DL2000 DNA marker; lanes P1, P2, P6, P10, some of the generations of rL-hIFN- $\lambda$.

Growth characteristics of the recombinant virus. To compare the growth characteristics of the recombinant viruses rL-hIFN- $\lambda 1$ and NDV, viral titers of the supernatants collected from infected SGC cells at $0,24,36,48$ and $72 \mathrm{~h}$ were determined by $\mathrm{TCID}_{50}$. The growth properties of $\mathrm{rL}-\mathrm{hIFN}-\lambda 1$ in the SGC cells were similar to those of the parental virus NDV (Fig. 2A). The result demonstrated that the recombinant viruses had mostly retained the growth characteristics of the parental virus in the SGC cells.

The allantoic cavities of 10-day-old embryonated SPF chicken eggs were inoculated with $10^{4} \mathrm{TCID}_{50}$ of rL-hIFN- $\lambda 1$. The allantoic fluid was harvested after 4 -day incubation. The viral titers was determined in duplicate by end-point titration. The results of the replication of $\mathrm{rL}-\mathrm{hIFN}-\lambda 1$ indicated that the amount of the recombinant virus remains similar after 10 passages (Fig. 2B). After 10 passages, the presence of the human IFN- $\lambda 1$ gene was confirmed by RT-PCR (Fig. 2C) and nucleotide sequence analysis (data not shown).

Human gastric carcinoma cell lines and human peripheral blood mononuclear cells express IFN- $\lambda 1$ specific receptor. To determine the expression of IFN $-\lambda$ receptor specific receptor IFNLR1 in human gastric cancer cell lines. We used RT-PCR assays and western blot analysis to analyze mRNA and protein expression of IFNLR1, respectively. The IFNLR1 was expressed in all examined gastric cancer cell lines, while the expression level of mRNA or protein was divided vividly among cell lines (Fig. 3), the SGC and AGS gastric cells expressed more IFNLR1 compared with other gastric tumor cells. For this reason we chose these two cell lines for our further study. The IFN- $\lambda$ receptor IFNLR1 expressed in gastric cancer cell lines showed potential as a target for IFN- $\lambda 1$ antitumor therapy. We found that PBMCs (human peripheral blood mononuclear cells) also expressed IFNLR1, which allowed us to study the antitumor immune response of rL-hIFN- $\lambda 1$.

Expression of IFN- $\lambda 1$ by $r L-h I F N-\lambda 1$-infected gastric adenocarcinoma cells. To define whether the IFN- $\lambda 1$ gene integrated in the NDV genome, the infected gastric tumor cell lines SGC were tested for production of the protein IFN- $\lambda 1$. The presence of IFN- $\lambda 1$ genes in the viral genome and expression in virus-infected cells were confirmed as positive for all passage generations using indirect immunofluorescence (Fig. 4A) and western blot analysis (Fig. 4B). In the indirect immunofluorescence the IFN- $\lambda 1$ protein (green) was highly expressed in the rL-hIFN- $\lambda 1$-infected group, while the NDV protein (red) was expressed in both the rL-hIFN- $\lambda 1$-infected group and NDV-infected group. Neither IFN- $\lambda 1$ nor NDV protein was expressed in the control group. 
A

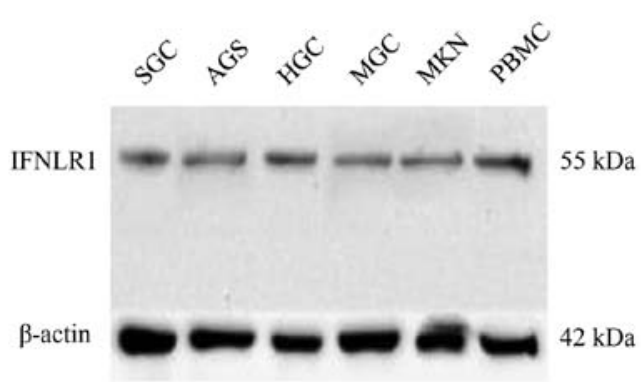

B

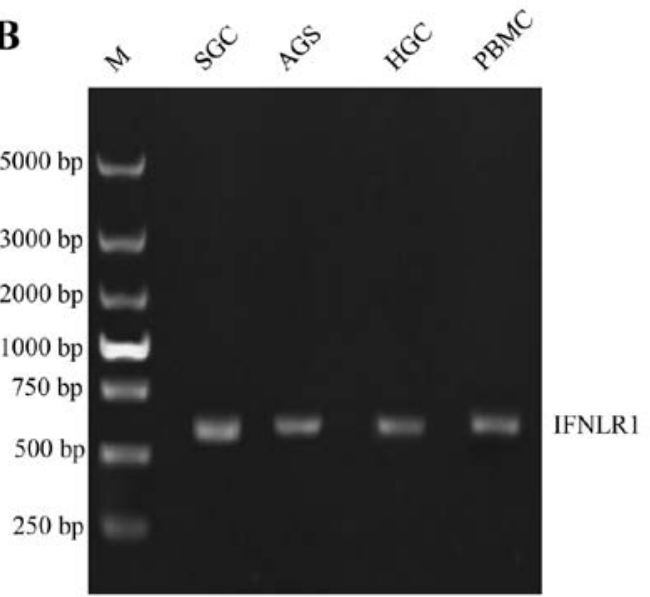

Figure 3. Human gastric cell lines express interferon- $\lambda 1$ (IFN- $\lambda 1$ 1) receptor IFNLR1. (A) The expression of IFNLR1 was measured by western blot analysis in a panel of five human gastric tumor cell lines and human PBMCs. (B) mRNA expression of IFNLR1 was evaluated by RT-PCR for the expression of IFNLR1 on three gastric tumor cell lines SGC, AGS and HGC and on PBMCs.

A

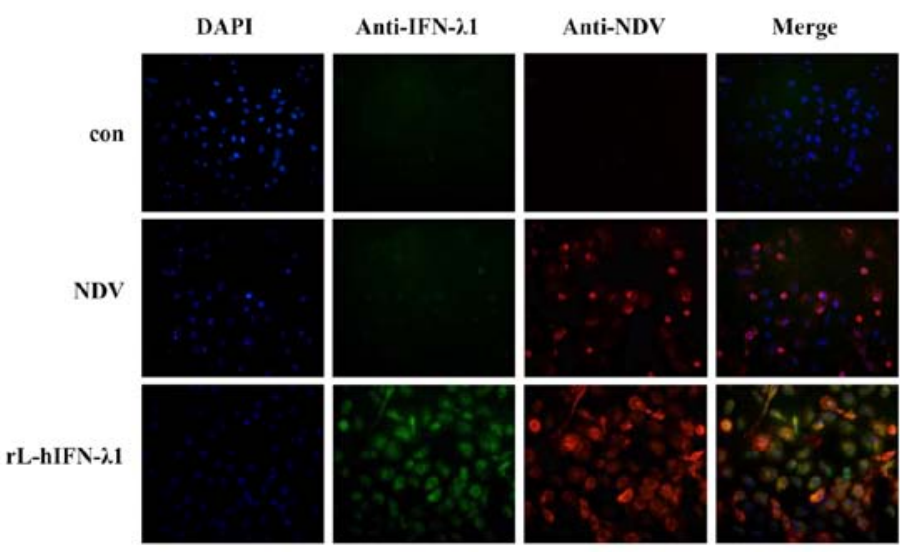

C

B

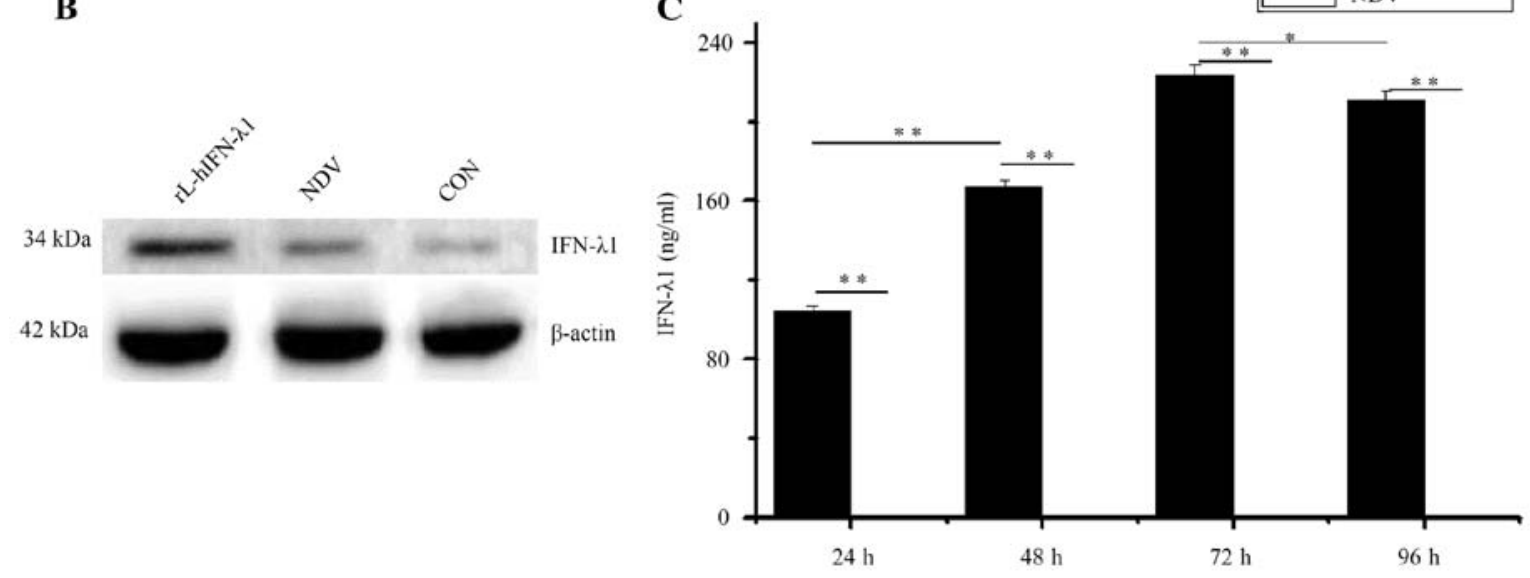
를

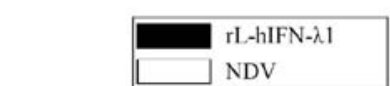

Figure 4. Expression of viral genes and proteins in infected gastric adenocarcinoma cells. (A) Indirect immunofluorescence staining of human interferon- $\lambda 1$ recombinant adenovirus (rL-hIFN- $\lambda 1$ )-infected SGC cells with chicken anti-Newcastle disease virus (NDV) serum and anti-IFN- $\lambda 1$ antibody observed with immunofluorescence microscope (x200 magnification). (B) IFN- $\lambda 1$ protein expression in SGC cells was detected by western blot analysis. The IFN- $\lambda 1$ protein was highly present in the rL-hIFN- $\lambda 1$-infected group compared with NDV group and control group. (C) SGC tumor cells were infected with rL-hIFN- $\lambda 1$ or NDV for $1 \mathrm{~h}$ and washed, After culture for $24,48,72$ and $96 \mathrm{~h}$, the supernatants were collected and analyzed for IFN- $\lambda 1$ by ELISA; ${ }^{*} \mathrm{p}<0.05,{ }^{* *} \mathrm{p}<0.01$.

To define whether the IFN- $\lambda 1$ gene integrated in the NDV genome leads to the production of biologically active IFN- $\lambda 1$ in supernatants of $\mathrm{rL}-\mathrm{hIFN}-\lambda 1$-infected gastric tumor cells, we measured the quantitation of IFN- $\lambda 1$ by ELISA. The supernatants of tumour cells were harvested 24,
48,72 and $96 \mathrm{~h}$ after the infection. The IFN- $\lambda 1$ expression reached the highest level at $72 \mathrm{~h}$ to $223 \mathrm{ng} / \mathrm{ml}$ in the supernatants of rL-hIFN- $\lambda 1$-infected SGC cells, whereas the SGC cells infected with NDV did not release any IFN- $\lambda 1$ into the supernatants (Fig 4C). 


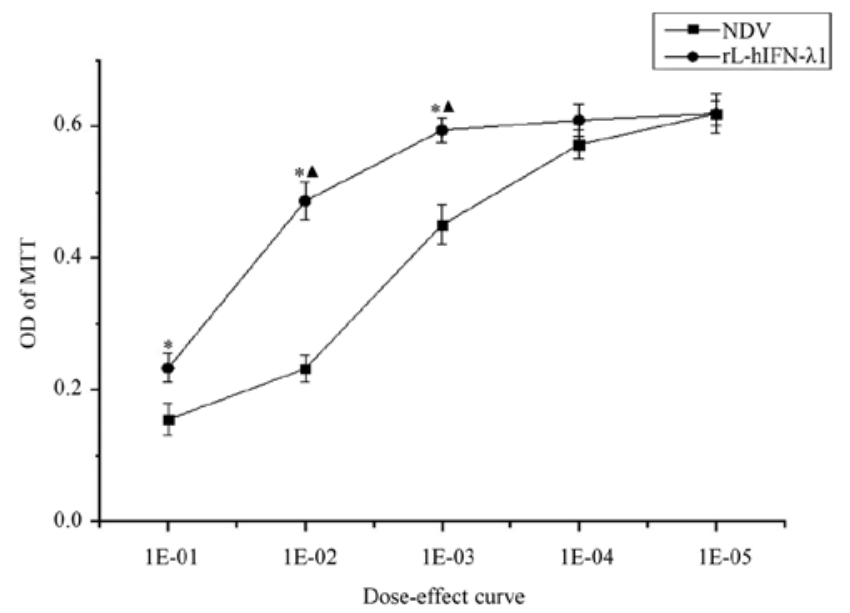

Figure 5. Proliferation changes in SGC cells after viral infection. Dose-response curve of SGC cells after viral infection at $24 \mathrm{~h}$. The OD of MTT after dilution $(n=5)$. The cells infected with human interferon- $\lambda 1$ recombinant adenovirus (rL-hIFN- $\lambda 1$ ) for $24 \mathrm{~h}$ showed significantly decreased absorbance when compared with OD of the Newcastle disease virus (NDV)-infected group ( $\left.{ }^{*} \mathrm{p}<0.05\right)$. A significant difference was observed between different dilutions of the same virus $\left({ }^{\wedge} \mathrm{p}<0.05\right)$.

The proliferation changes of $S G C$ cells infected with $r L-h I F N-\lambda 1$. To elucidate the proliferation changes of rL-hIFN- $\lambda 1$-infected gastric adenocarcinoma cells, MTT assay was utilized to portray dose-response curves. After $24 \mathrm{~h}$ of $\mathrm{rL}-\mathrm{hIFN}-\lambda 1$ or NDV infection, the gastric tumour cells were assessed by MTT assay. OD of MTT in the rL-hIFN- $\lambda 1$-infected group was weaker compared with that in the NDV-infected group, suggested that the rL-hIFN- $\lambda 1$-infected group had a greater inhibition ratio. Moreover, the inhibition ratio increased over the dose after infection (Fig. 5). $r L-h I F N-\lambda I$ induces apoptosis in gastric tumor cells. The recombinant virus rL-hIFN- $\lambda 1$ was designed for cancer therapeutics, and apoptosis was measured in gastric carcinoma cell lines.

TUNEL assay kit was used for monitoring apoptosis. The results revealed that the number of apoptotic cells and the AI was much higher in the rL-hIFN- $\lambda 1$ compared with NDV group, the negative control group showed much less apoptosis $(\mathrm{p}<0.01)$ (Fig. 6A).

Furthermore, the levels of cleaved caspase-3, a key protein to indicate apoptosis, were measured by western blot analysis at $24 \mathrm{~h}$ after virus infecting tumour cell lines. The expression levels of cleaved caspase- 3 proteins were upregulated in the rL-hIFN- $\lambda 1$ group compared with the other NDV group and the negative control group (Fig. 6B).

Flow cytometry analysis showed that early apoptosis of cells was higher in the rL-hIFN- $\lambda 1$-infecting group than that in NDV group, early apoptosis was not detected in the control group (Fig. 6C).

$r L$-hIFN- $\lambda 1$ modulates the human Th1/Th2 response in the tumor microenvironment. Th1/Th2 balance is confirmed to play an important role in tumor growth, the high expression of Th1 response and reduction is capable of inducing antitumor immunity to prevent cancer cell lines in preclinical study $(14,27)$ and gastric cancer in clinical research (28). To determine whether rL-hIFN- $\lambda 1$ could elevate the secretion of chemokines of tumor microenvironment to promote antitumor immune responses, SGC cells were infected with $\mathrm{LL}-\mathrm{hIFN}-\lambda 1$ or NDV, after cultured for $72 \mathrm{~h}$, supernatants were collected to stimulate PBMCs for 5 days, then the production as quantified of T-cell cytokines IFN- $\gamma$ (representative of Th1 responses) and IL-13 (representative of Th2 responses) by ELISA everyday. Our studies revealed that the Th1 cytokine IFN- $\gamma$ increased significantly and the Th 2 cytokine IL-13 was highly decreased
A

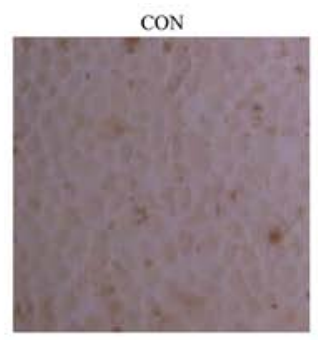

C

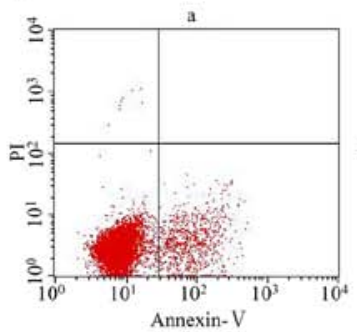

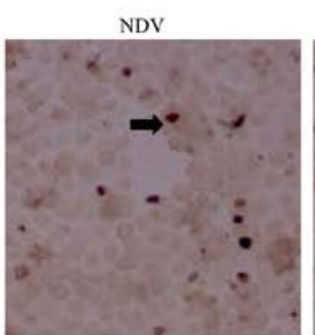
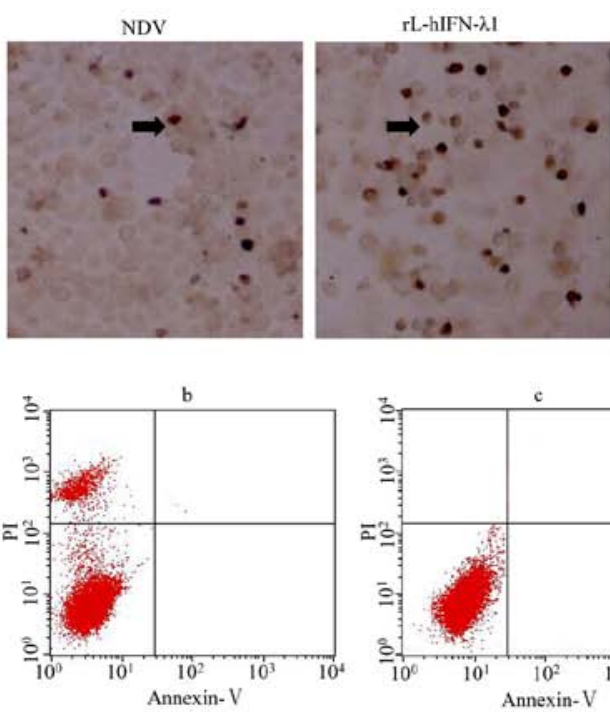
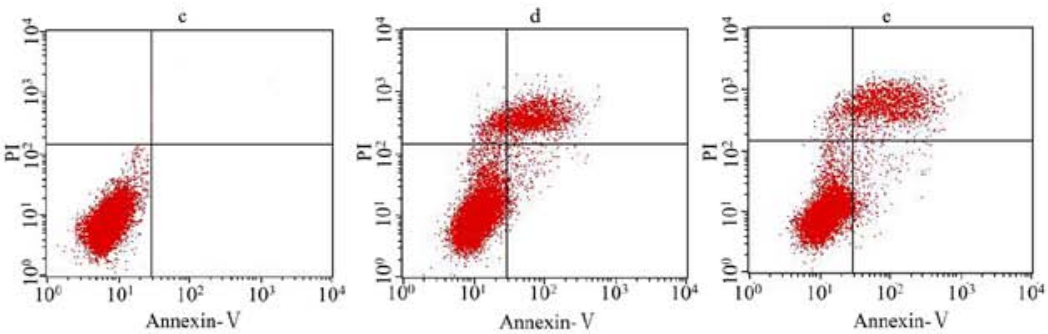

Figure 6. Apoptosis in SGC cells at $24 \mathrm{~h}$ after infection. (A) TUNEL analysis of apoptosis in SGC cells. Apoptosis of virus-infected cells was obvious compared with the controls. The apoptotic cells $(\rightarrow)$ in the human interferon- $\lambda 1$ recombinant adenovirus (rL-hIFN- $\lambda 1$ ) groups were more abundant compared with those in the Newcastle disease virus (NDV) group. (B) The expression of apoptosis-associated proteins in SGC cells was detected by western blot analysis. The protein cleaved caspase- 3 had higher expression levels in rL-hIFN- $\lambda 1$ group compared with those in the NDV group and control group. (C) Flow cytometry analysis results of $24 \mathrm{~h}$ early apoptosis cells. (C-a) Annexin V positive control. (C-b) Propidium iodide (PI) positive control. (C-c) SGC cells without treating with virus as a negative control. (C-d) SGC cells were infected with NDV, the apoptotic rate produced by NDV was $9.43 \%$. (C-e) SGC cells were infected with $\mathrm{rL}-\mathrm{hIFN}-\lambda 1$, the apoptotic rate produced by $\mathrm{rL}-\mathrm{hIFN}-\lambda 1$ was $11.07 \%$. 
A
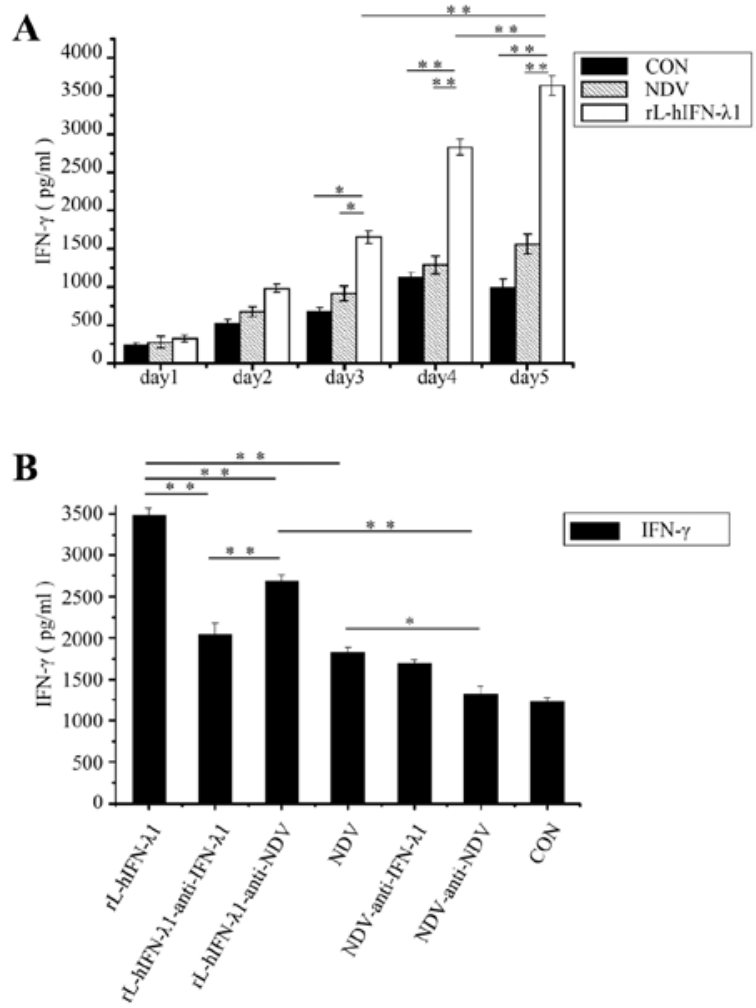
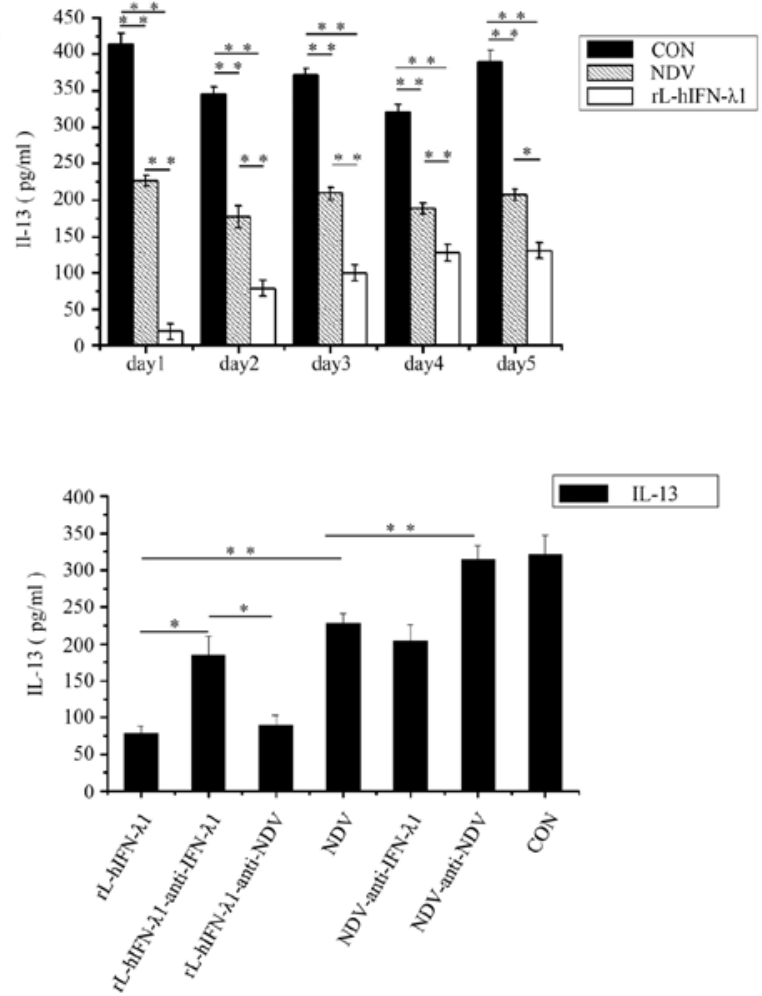

Figure 7. Effect of human interferon- $\lambda 1$ recombinant adenovirus (rL-hIFN- $\lambda 1$ ) on T helper cell subtype 1 (Th1) (IFN- $\gamma$ ) and Th2 [interleukin (IL)-13] production by Con-A-stimulated PBMCs. (A) PBMCs stimulated with Con-A $(5.0 \mathrm{mg} / \mathrm{ml})$ were co-cultured for $1-5$ days with the supernatants of SGC cells collected after 3 days infection with rL-hIFN- $\lambda 1$ or NDV. The cytokine protein levels were measured by ELISA. (B) Anti-IFN- $\lambda 1$ (anti-29) or anti-NDV was added into the former supernatants collected as described to detect whether the virus itself or the cytokine protein produced by the viral infection tumor cells played a dominant role in the changing of Th1/Th2 immuno-response. The left panel shows a significant decrease in the expression of IFN- $\gamma$ in the anti-IFN- $\lambda 1$ added group compared with group added with anti-NDV. The right panel showed that the IL-13 has a higher increase in anti-IFN- $\lambda 1$ added group than the anti-NDV group $\left({ }^{*} \mathrm{p}<0.05\right)\left({ }^{* *} \mathrm{p}<0.01\right)$

in the rL-hIFN- $\lambda 1$ group, as compared with NDV and control groups $(\mathrm{p}<0.01)$ (Fig. 7A). To confirm whether the Th1/Th2 immune response change was related to cytokine IFN- $\lambda 1$ or the NDV viral, anti-IFN- $\lambda 1$ or anti-NDV was added during the cell culture with the former supernatants, then cytokine levels were tested after PBMCs cultured for 5 days. The Th1 response was enhanced, and the Th2 response inhibited much more in the anti-IFN- $\lambda 1$ group than in the anti-NDV group, NDV group and control group showed no significant change. The results demonstrated that the tumor microenvironment cytokine skewed towards a Th1 response duo to the high dose of IFN- $\lambda 1$ expressed by the rL-hIFN- $\lambda 1$-infected tumour cells (Fig. 7B).

\section{Discussion}

Oncolytic viral therapy is a method that harnesses the natural ability of a virus to infect, duplicate and lyse a host cell as part of its natural life cycle (29). NDV, an oncolytic virus, has been confirmed to possess the capablity to inhibit malignant cells via multiple mechanisms (30). In recent years, NDV has been shown to induce the immune system to eliminate tumor cells (31), and NDV strains can selectively replicate up to 10,000 times better in tumor cells than in normal cells (32). With the development of reverse genetics, modification of the viral genome for NDV as well as introduction of foreign sequences has become possible (33).
IFN- $\lambda 1$ (IL-29), a recently discovered cytokine, accompanies type I IFN in the signaling pathways and differs wildly in tissue responsiveness. It is known that the epithelial cells of most tissues express IFNLR1 and are responsive to IFN- $\lambda$ in mice $(34,35)$. Most of the tumor cells are epithelial in origin. The tumor cells express IFNLR1 and are responsive to IFN- $\lambda 1$. On the other hand, IFN- $\lambda 1$ receptor also expressed in both naive and memory $\mathrm{CD}^{+} \mathrm{T}$ cells (22). $\mathrm{T}$ cells are capable of responding to IFN- $\lambda 1$ with altering cytokine production, it allows IFN- $\lambda 1$ to alter the balance of Th1 and Th2 immune responses, which could be potential agents for cancer therapy $(27,36,37)$. IFN- $\lambda 1$ has also been shown to exert antitumor effects in both murine and human models (24).

Due to the above advantages of tumour therapy, we inserted human IFN- $\lambda 1$ gene into the NDV viral genome, in such a way that the viral infection of tumor cells leads to the expression of IFN- $\lambda 1$. We propose that the recombinant virus rL-hIFN- $\lambda 1$ may have antitumor therapy potential. At the start of this study, there was no study on genetically engineering recombined NDV expressing IFN- $\lambda 1$ genes to improve the effect of cancer therapy.

In the present study, we confirmed the stability of growth characteristics of our recombinant virus was as much as the NDV virus passing 10 generations in the embryonated SPF eggs. The results have also shown the high dose stable expression of IFN- $\lambda 1$ gene in $\mathrm{rL}-\mathrm{hIFN}-\lambda 1$ infected tumour cell lines at $24 \mathrm{~h}$ reaching the highest levels at $72 \mathrm{~h}$. 
In recent years, some studies have confirmed apoptosis as the dominant key to NDV-related cell death (29,38-41). It was demonstrated in our previous study that NDV caused gastric adenocarcinoma SGC-7901 and AGS cell death via apoptosis (42). The mechanism behind NDV oncolytic effect have been investigated. It was recently found that its apoptosis inducing effect is presumably exerted through ER-mediated cellular stress mechanism (43-45). Our previous study also found in addition to apoptosis, that NDV can also induce autophagy, ERs and mitochondrial dysfunction (42).

The new generated recombinant NDV LaSota strain is designed as a potential candidate for a viral vector in cancer therapy in human, we further found that the high levels of IFN- $\lambda 1$ receptor IFNLR 1 was expressed in the gastric tumor cell lines SGC and AGS, which indicated that gastric cancer could be a potential target for IFN- $\lambda 1$ and rL-hIFN- $\lambda 1$ therapy. The TUNEL assay, western blot assay and the Annexin V flow cytometric analysis all confirmed that the number of apoptotic cells and the AI were markedly higher in the rL-hIFN- $\lambda 1$ and NDV groups compared with control group, and $\mathrm{rL}-\mathrm{hIFN}-\lambda 1$ group exhibited a higher AI compared with NDV groups.

The high dose of cytokine expressed by the rL-hIFN- $\lambda 1$ infecting tumour cells had pro-tumor Th2 immune responses and in favor of antitumor Th1 responses in PBMCs, which generate an antitumour environment making the rL-hIFN- $\lambda 1$ a protential antitumor agent for immunotherapeutic approaches.

In conclusion, $\mathrm{rL}-\mathrm{hIFN}-\lambda 1$ inhibited the growth of gastric cancer cell lines which contained the IFN $\lambda$-R1 receptors and accelerated apoptosis to a certain extent. The recombinant rL-hIFN- $\lambda 1$ virus modulated Th1/Th 2 immune response to change the tumor microenvironment to an antitumour cytokine which led to the reducing of tumor growth. The present study is expected to provide an experimental basis for further mechanism research and clinical application of $\mathrm{rL}-\mathrm{hIFN}-\lambda 1$ in gastric adenocarcinoma therapy, considered that the recombinant NDV LaSota expressing human IFN- $\lambda 1$ named as rL-hIFN- $\lambda 1$ could be a potential viral agent for gastric tumor therapy.

\section{Acknowledgements}

The authors would like to thank Zhijian Zhang and Ai-Hua Gong from Jiangsu University (Jiangsu, China) for kindly providing suggestions of the experiments performed. We thank all our laboratory members for their help. The present study was supported by the Natural Science Foundation of Jiangsu Province (grant no. BK20151333) and the Social Development Fund of Zhenjiang, China (SH2014046).

\section{References}

1. Siegel RL, Miller KD and Jemal A: Cancer statistics, 2015. CA Cancer J Clin 65: 5-29, 2015.

2. Khalighinejad N, Hariri H, Behnamfar O, Yousefi A and Momeni A: Adenoviral gene therapy in gastric cancer: A review. World J Gastroenterol 14: 180-184, 2008.

3. Janke M, Peeters B, de Leeuw O, Moorman R, Arnold A, Fournier P and Schirrmacher V: Recombinant Newcastle disease virus (NDV) with inserted gene coding for GM-CSF as a new vector for cancer immunogene therapy. Gene Ther 14: 1639-1649, 2007.
4. Beutner U, Lorenz U, Illert B, Rott L, Timmermann W, Vollmers HP, Müller-Hermelink HK, Thiede A and Ulrichs K: Neoadjuvant therapy of gastric cancer with the human monoclonal IgM antibody SC-1: Impact on the immune system. Oncol Rep 19: 761-769, 2008.

5. Krishnamurthy S and Samal SK: Nucleotide sequences of the trailer, nucleocapsid protein gene and intergenic regions of Newcastle disease virus strain Beaudette $C$ and completion of the entire genome sequence. J Gen Virol 79: 2419-2424, 1998.

6. Bukreyev A and Collins PL: Newcastle disease virus as a vaccine vector for humans. Curr Opin Mol Ther 10: 46-55, 2008.

7. Bukreyev A, Skiadopoulos MH, Murphy BR and Collins PL: Nonsegmented negative-strand viruses as vaccine vectors. J Virol 80: 10293-10306, 2006.

8. Ge J, Deng G, Wen Z, Tian G, Wang Y, Shi J, Wang X, Li Y, Hu S, Jiang Y, et al: Newcastle disease virus-based live attenuated vaccine completely protects chickens and mice from lethal challenge of homologous and heterologous $\mathrm{H} 5 \mathrm{~N} 1$ avian influenza viruses. J Virol 81: 150-158, 2007.

9. Ge J, Tian G, Zeng X, Jiang Y, Chen H and Bua Z: Generation and evaluation of a Newcastle disease virus-based H9 avian influenza live vaccine. Avian Dis 54 (Suppl 1): 294-296, 2010.

10. Elliott S, Egrie J, Browne J, Lorenzini T, Busse L, Rogers N and Ponting I: Control of rHuEPO biological activity: The role of carbohydrate. Exp Hematol 32: 1146-1155, 2004.

11. Sinclair AM and Elliott S: Glycoengineering: The effect of glycosylation on the properties of therapeutic proteins. J Pharm Sci 94: 1626-1635, 2005.

12. Sheppard P, Kindsvogel W, Xu W, Henderson K, Schlutsmeyer S, Whitmore TE, Kuestner R, Garrigues U, Birks C, Roraback J, et al: IL-28, IL-29 and their class II cytokine receptor IL-28R. Nat Immunol 4: 63-68, 2003.

13. Sommereyns C, Paul S, Staeheli P and Michiels T: IFN-lambda (IFN-lambda) is expressed in a tissue-dependent fashion and primarily acts on epithelial cells in vivo. PLoS Pathog 4: e1000017, 2008.

14. Haabeth OA, Lorvik KB, Hammarström C, Donaldson IM, Haraldsen G, Bogen B and Corthay A: Inflammation driven by tumour-specific Th1 cells protects against B-cell cancer. Nat Commun 2: 240, 2011.

15. Mohty AM, Grob JJ, Mohty M, Richard MA, Olive D and Gaugler B: Induction of IP-10/CXCL10 secretion as an immunomodulatory effect of low-dose adjuvant interferon-alpha during treatment of melanoma. Immunobiology 215: 113-123, 2010.

16. Pertl U, Luster AD, Varki NM, Homann D, Gaedicke G, Reisfeld RA and Lode HN: IFN-gamma-inducible protein-10 is essential for the generation of a protective tumor-specific CD8 $\mathrm{T}$ cell response induced by single-chain IL-12 gene therapy. J Immunol 166: 6944-6951, 2001.

17. Tominaga M, Iwashita Y, Ohta M, Shibata K, Ishio T, Ohmori N, Goto T, Sato S and Kitano S: Antitumor effects of the MIG and IP-10 genes transferred with poly [D,L-2,4-diaminobutyric acid] on murine neuroblastoma. Cancer Gene Ther 14: 696-705, 2007.

18. Wang P, Yang X, Xu W, Li K, Chu Y and Xiong S: Integrating individual functional moieties of CXCL10 and CXCL11 into a novel chimeric chemokine leads to synergistic antitumor effects: A strategy for chemokine-based multi-target-directed cancer therapy. Cancer Immunol Immunother 59: 1715-1726, 2010.

19. Egeter O, Mocikat R, Ghoreschi K, Dieckmann A and Röcken M: Eradication of disseminated lymphomas with CpG-DNA activated T helper type 1 cells from nontransgenic mice. Cancer Res 60: 1515-1520, 2000.

20. Müller-Hermelink N, Braumüller H, Pichler B, Wieder T, Mailhammer R, Schaak K, Ghoreschi K, Yazdi A, Haubner R, Sander CA, et al: TNFR1 signaling and IFN-gamma signaling determine whether $\mathrm{T}$ cells induce tumor dormancy or promote multistage carcinogenesis. Cancer Cell 13: 507-518, 2008.

21. Shankaran V, Ikeda H, Bruce AT, White JM, Swanson PE, Old LJ and Schreiber RD: IFNgamma and lymphocytes prevent primary tumour development and shape tumour immunogenicity. Nature 410: 1107-1111, 2001.

22. Dai J, Megjugorac NJ, Gallagher GE, Yu RY and Gallagher G: IFN-lambda1 (IL-29) inhibits GATA3 expression and suppresses Th2 responses in human naive and memory T cells. Blood 113: 5829-5838, 2009.

23. Sargurupremraj M, Pukelsheim K, Hofer T and Wjst M: Intermediary quantitative traits - an alternative in the identification of disease genes in asthma? Genes Immun 15: 1-7, 2014.

24. Stiff A and Carson Iii W: Investigations of interferon-lambda for the treatment of cancer. J Innate Immun 7: 243-250, 2015. 
25. Bu X, Wang M, Zhang J, Liu J, Jia L, Liang B and Yan Y: Recombinant adenovirus expressing hIFN- $\lambda 1$ inhibits gastric adenocarcinoma cell line SGC-7901 proliferation. Oncol Lett 11: 287-292, 2016

26. Wyatt LS, Moss B and Rozenblatt S: Replication-deficient vaccinia virus encoding bacteriophage T7 RNA polymerase for transient gene expression in mammalian cells. Virology 210: 202-205, 1995

27. Burkart C, Arimoto K, Tang T, Cong X, Xiao N, Liu YC, Kotenko SV, Ellies LG and Zhang DE: Usp18 deficient mammary epithelial cells create an antitumour environment driven by hypersensitivity to IFN- $\lambda$ and elevated secretion of $\mathrm{Cxcl} 10$. EMBO Mol Med 5: 967-982, 2013.

28. Reichard KW, Lorence RM, Cascino CJ, Peeples ME, Walter RJ, Fernando MB, Reyes HM and Greager JA: Newcastle disease virus selectively kills human tumor cells. J Surg Res 52: 448-453, 1992.

29. Foucault C, Mordant P, Grand B, Achour K, Arame A, Dujon A, Le Pimpec Barthes F and Riquet M: Unexpected extensions of non-small-cell lung cancer diagnosed during surgery: Revisiting exploratory thoracotomies and incomplete resections. Interact Cardiovasc Thorac Surg 16: 667-672, 2013.

30. Vähä-Koskela MJ, Heikkilä JE and Hinkkanen AE: Oncolytic viruses in cancer therapy. Cancer Lett 254: 178-216, 2007.

31. Hossain A, Radwan FF, Doonan BP, God JM, Zhang L, Bell PD and Haque A: A possible cross-talk between autophagy and apoptosis in generating an immune response in melanoma. Apoptosis 17: 1066-1078, 2012.

32. Lam HY, Yeap SK, Rasoli M, Omar AR, Yusoff K, Suraini AA and Alitheen NB: Safety and clinical usage of Newcastle disease virus in cancer therapy. J Biomed Biotechnol 2011: $718710,2011$.

33. Nakaya T, Cros J, Park MS, Nakaya Y, Zheng H, Sagrera A, Villar E, García-Sastre A and Palese P: Recombinant Newcastle disease virus as a vaccine vector. J Virol 75: 11868-11873, 2001.

34. Lasfar A, Abushahba W, Balan M and Cohen-Solal KA: Interferon lambda: A new sword in cancer immunotherapy. Clin Dev Immunol 2011: 349575, 2011.

35. Steen HC and Gamero AM: Interferon-lambda as a potential therapeutic agent in cancer treatment. J Interferon Cytokine Res 30: 597-602, 2010.
36. Jordan WJ, Eskdale J, Srinivas S, Pekarek V, Kelner D, Rodia $M$ and Gallagher G: Human interferon lambda-1 (IFN-lambda1/IL-29) modulates the Th1/Th2 response. Genes Immun 8: 254-261, 2007.

37. Ding S, Khoury-Hanold W, Iwasaki A and Robek MD: Epigenetic reprogramming of the type III interferon response potentiates antiviral activity and suppresses tumor growth. PLoS Biol 12: e1001758, 2014.

38. Elankumaran S, Rockemann D and Samal SK: Newcastle disease virus exerts oncolysis by both intrinsic and extrinsic caspase-dependent pathways of cell death. J Virol 80: 7522-7534, 2006.

39. Hrabák A, Csuka I, Bajor T and Csatáry LK: The cytotoxic anti-tumor effect of MTH-68/H, a live attenuated Newcastle disease virus is mediated by the induction of nitric oxide synthesis in rat peritoneal macrophages in vitro. Cancer Lett 231: 279-289, 2006.

40. Bian H,Fournier P, Peeters B and Schirrmacher V: Tumor-targeted gene transfer in vivo via recombinant Newcastle disease virus modified by a bispecific fusion protein. Int J Oncol 27: 377-384, 2005.

41. Lorence RM, Rood PA and Kelley KW: Newcastle disease virus as an antineoplastic agent: Induction of tumor necrosis factor-alpha and augmentation of its cytotoxicity. J Natl Cancer Inst 80: 1305-1312, 1988 .

42. Bu XF, Wang MB, Zhang ZJ, Zhao YH, Li M and Yan YL: Autophagy is involved in recombinant Newcastle disease virus (rL-RVG)-induced cell death of stomach adenocarcinoma cells in vitro. Int J Oncol 47: 679-689, 2015.

43. Fábián Z, Töröcsik B, Kiss K, Csatary LK, Bodey B, Tigyi J, Csatary C and Szeberényi J: Induction of apoptosis by a Newcastle disease virus vaccine (MTH-68/H) in PC12 rat phaeochromocytoma cells. Anticancer Res 21 (1A): 125-135, 2001.

44. Fábián Z, Csatary CM, Szeberényi J and Csatary LK: p53-independent endoplasmic reticulum stress-mediated cytotoxicity of a Newcastle disease virus strain in tumor cell lines. J Virol 81: 2817-2830, 2007.

45. Fábián Z, Vecsernyés M, Pap M and Szeberényi J: The effects of a mutant $\mathrm{p} 53$ protein on the proliferation and differentiation of PC12 rat phaeochromocytoma cells. J Cell Biochem 99: 1431-1441, 2006. 